\title{
Injection System of the SSC Medium Energy Booster
}

\author{
N. Mao, R. Gerig, and J. McGill \\ Superconducting Super Collider Laboratory* \\ 2550 Beckleymeade Ave. \\ Dallas, TX 75237, USA
}

K. Brown

Stanford Linear Accelerator Center
Stanford University
Stanford, CA 94309

April 1994

* Operated by the Universities Research Association, Inc., for the U.S. Department of Energy under Contract No. DE-AC35-89ER40486. 


\title{
Injection System of the SSC Medium Energy Booster
}

\author{
N. Mao, R. Gerig, J. McGill, and K. Brown
}

\begin{abstract}
The Medium Energy Booster (MEB) is the third of the SSCL accelerators and the largest of the resistive magnet synchrotrons. It accelerates protons from an injection momentum of $12 \mathrm{GeV} / \mathrm{c}$ to a top momentum of $200 \mathrm{GeV} / \mathrm{c}$. A beam injection system has been designed to inject the beam transferred from the Low Energy Booster (LEB) onto the MEB closed orbit in the MEB injection insertion region. The beam is injected via a vertical bending Lambertson septum magnet and a horizontal kicker with appropriate matching and very little beam loss and emittance dilution.

The beam optics of the injection system is described in this paper. The required parameters of the Lambertson septum magnet and the injection kicker are given.
\end{abstract}




\subsection{INTRODUCTION}

The MEB ring layout and the detail of the MEB injection insertion are shown in Figure $1 .^{1}$ The injection insertion adopts the standard FODO quadrupole spacing and uses missing dipoles to provide spaces for the beam transfer line, ${ }^{2}$ septum magnet and kicker. The injection philosophy is as follows. The septum magnet (SEP3, Figures 2 and 3), located right upstream of the focusing quadrupole Q152, bends the beam up by $2.173^{\circ}$ onto the MEB closed orbit plane. The horizontal kicker (HKICK), located right upstream of the focusing quadrupole Q154, completes the injection process, placing the beam onto the proper MEB horizontal closed orbit. This scheme takes advantage of the defocusing quadrupole Q153 present between the septum magnet and the horizontal kicker. The defocusing quadrupole bends the injection beam outward in the horizontal plane, thereby lessening the necessary strength of the injection kicker.

The lattice functions of the injection insertion are shown in Figure $4 .^{1}$ The functions at the exits of the septum magnet and the injection kicker are also listed in Table 1, lines 38 and 52, respectively. The injection system has been designed to inject the beam in both the collider fill mode and the test beam mode with the normalized transverse emittances ( $\mathrm{rms}$ ) of $0.6 \pi^{*} \mathrm{~mm} * \mathrm{mrad}$ and $4.0 \pi * \mathrm{~mm}^{*} \mathrm{mrad}$, respectively.

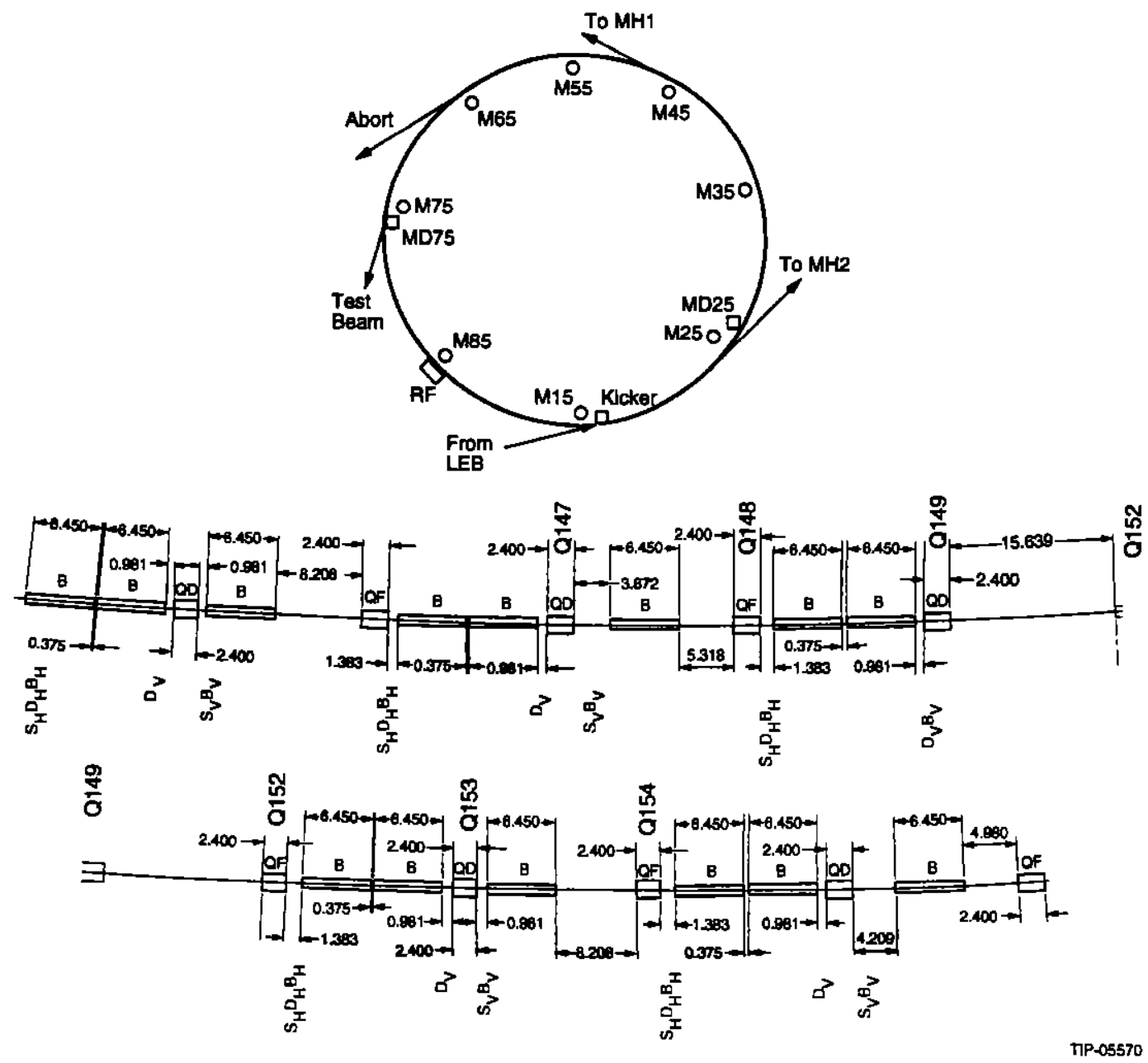

Figure 1. MEB RIng Layout and Injection Insertion. 


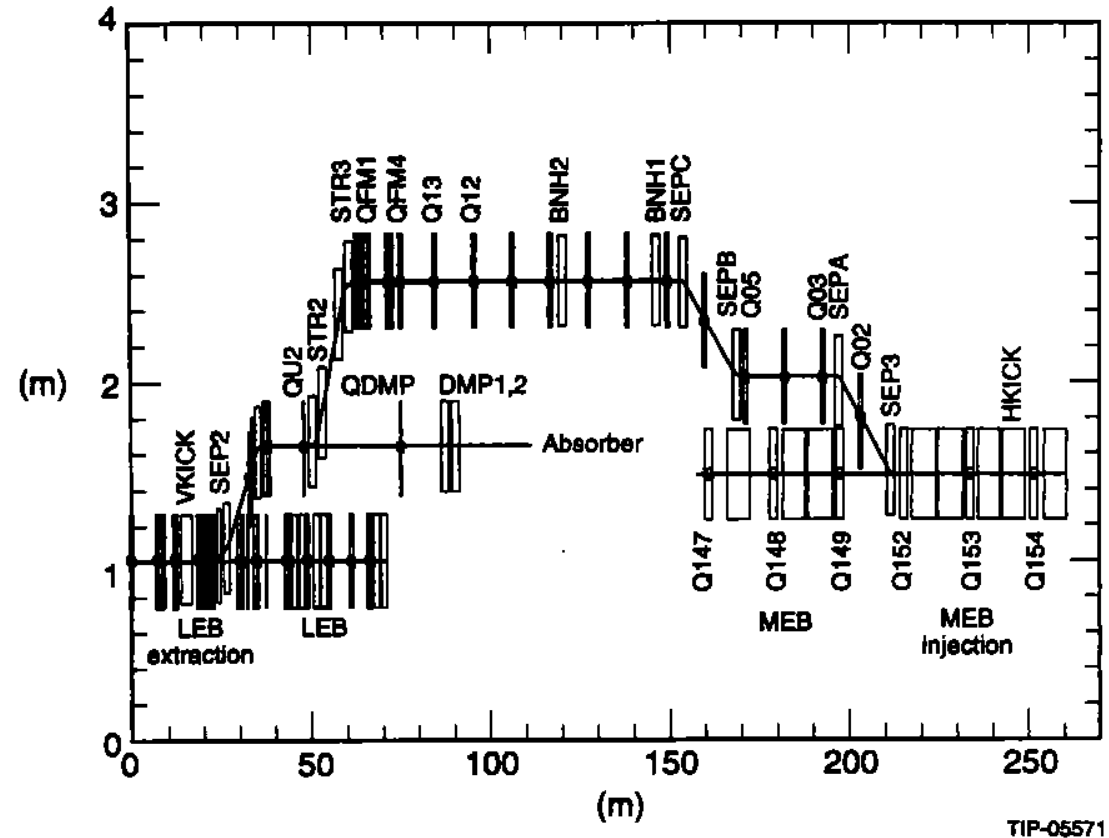

Figure 2. LEB-MEB Transfer Line and Absorber Line (Elevatlon Vlew, LEB630 and LEB917).

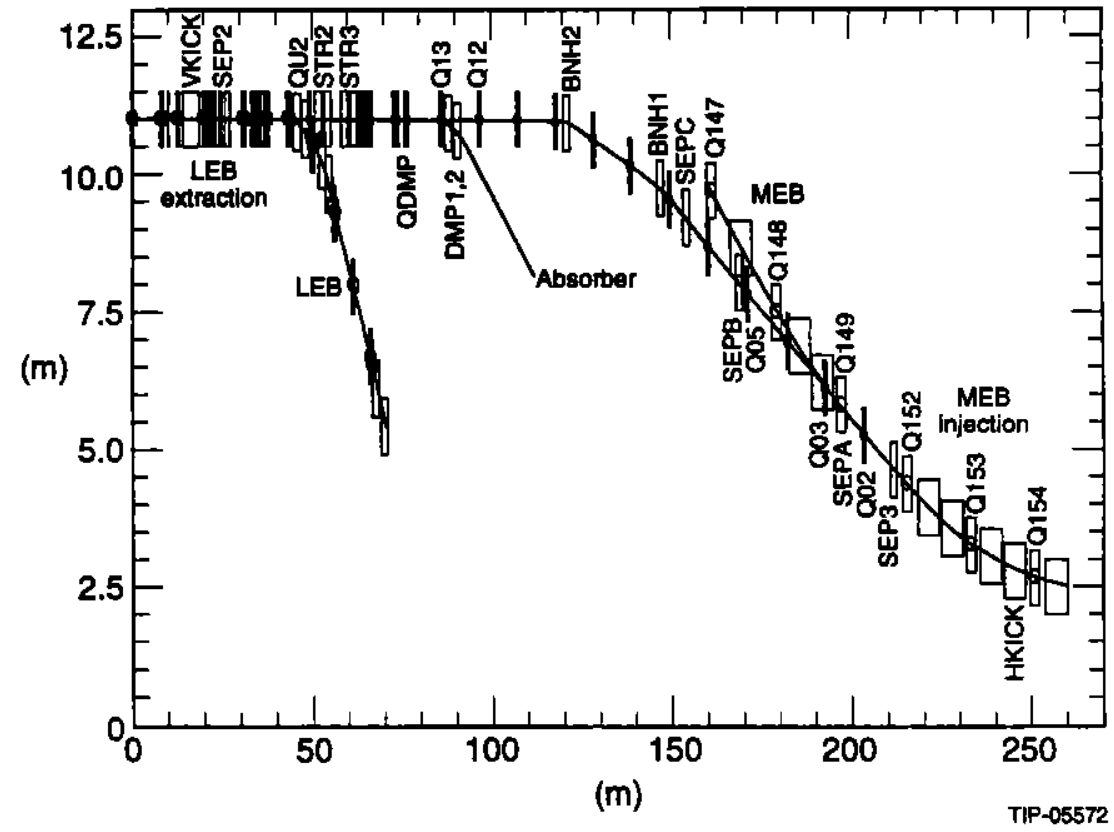

Figure 3. LEB-MEB Transfer Lne and Absorber LIne (Plan Vlew, LEB630 and LEB917). 
(a)

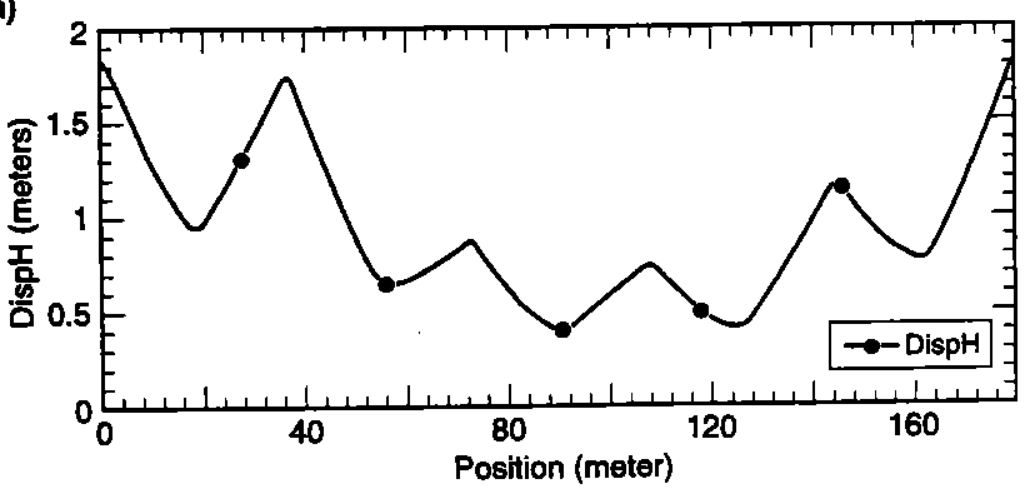

(b)

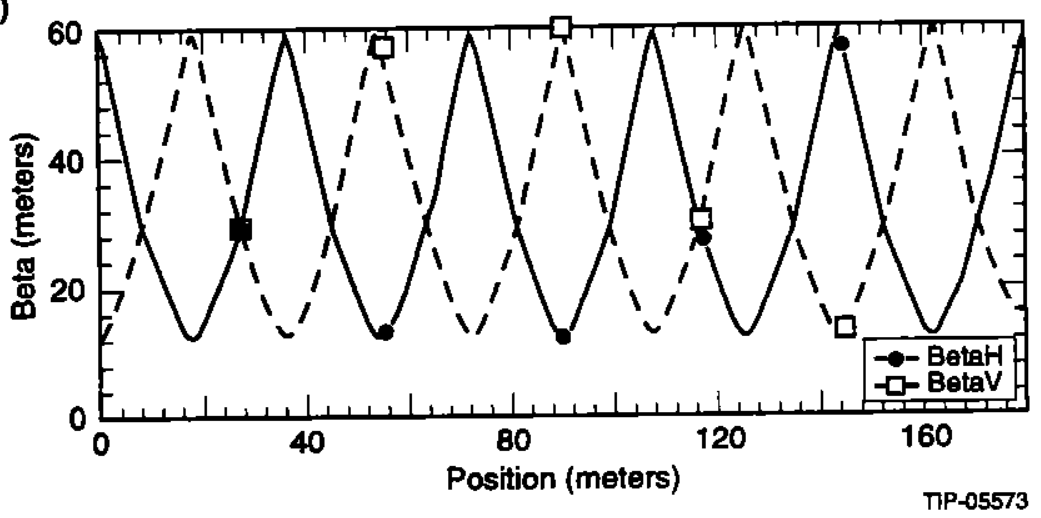

Figure 4. Lattice and Orblt Functlons for Injection Insertion. 


\subsection{BEAM OPTICS OF INJECTION SYSTEM}

The layout of the injection system is shown in Figure 5. The injection kicker (HKICK) is one cell downstream of the septum magnet (SEP3). The central trajectory separation between the injection beam and the circulating beam at the exit of the septum magnet in the horizontal plane is given by

$$
\Delta x=K \sqrt{\beta_{1} \beta_{2}} \sin \left(\phi_{2}-\phi_{1}\right)
$$

where $K$ is the kicker strength, $\beta_{1}$ and $\beta_{2}$ are the $\beta$ functions and $\phi_{2}$ and $\phi_{1}$ are the betatron phases at septum magnet exit and the kicker, respectively. Here the kicker is assumed to be a thin one. If the kicker has a length of $L$, the separation $\Delta x$ is given by

$$
\Delta x=\frac{K}{L} \sqrt{\beta_{1}} \int_{0}^{L} \sqrt{\beta_{2}(l)} \sin \left(\phi_{2}(l)-\phi_{1}\right) d l .
$$

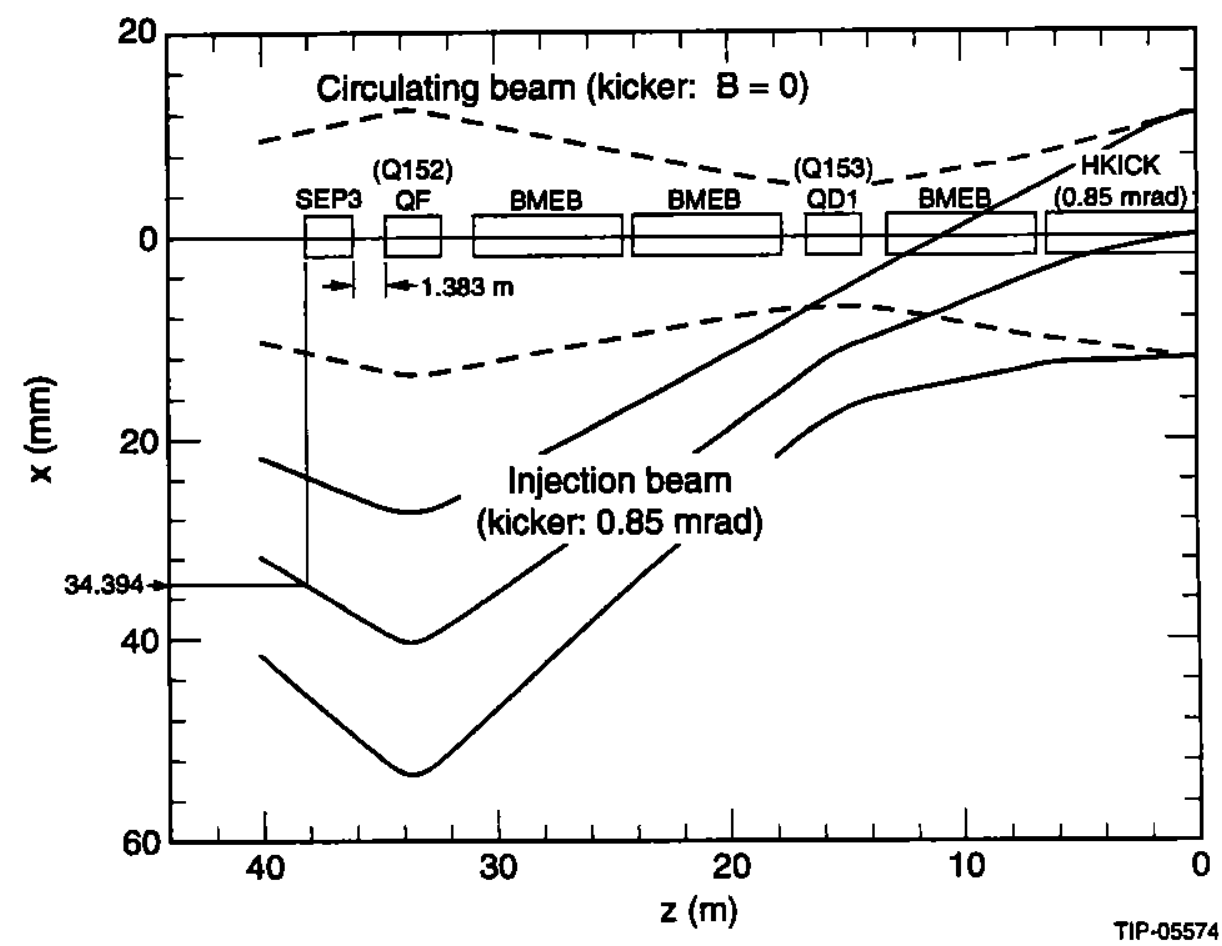

Figure 5. Layout of MEB Injection System. 
The requirement for the separation between the two beam centroids at the septum magnet is related to the sizes of two beams, the beam centroid displacements caused by the magnet errors, and the thickness of the septum magnet. The parameters used in the injection system design are as follows:

The normalized beam transverse emittance $\left(3 \sigma\right.$ test beam) $=9 * 4 \pi * \mathrm{~mm}^{*} \mathrm{mrad}$.

The $\beta$ and $\eta$ functions at the exit of the kicker, $\beta_{x}=51.0927 \mathrm{~m}$

$$
\begin{aligned}
& \beta_{y}=14.4093 \mathrm{~m} \\
& \alpha_{x}=-2.09588
\end{aligned}
$$$$
\alpha y=0.731054
$$$$
\eta_{\mathrm{x}}=-1.063048 \mathrm{~m}
$$$$
\eta_{\mathrm{y}}=0 \mathrm{~m}
$$$$
\eta_{\mathrm{x}}^{\prime}=-0.048624
$$$$
\eta_{y^{\prime}}=0 \text {. }
$$

The beam centroid displacement caused by the magnet errors $=3 \mathrm{~mm}$.

The thickness of the septum $=2 \mathrm{~mm}$.

Using these parameters, both analytical and numerical calculations show that a kicker strength of $0.85 \mathrm{mrad}$ is adequate. It corresponds to a separation of $34.394 \mathrm{~mm}$ between the two beam centroids at the entrance of the septum magnet. This amount of separation is large enough to provide a $2.0 \mathrm{~mm}$ space for the septum. The numerical calculation is carried out with code TRANSPORT. ${ }^{3}$ The layout of the injection system is shown in Figure 5; the beam parameters are shown in Figure 6 and explained as follows.

(1) Beam sizes $\left(3 \sigma, 4 \pi * \mathrm{~mm}^{*}\right.$ mrad test beam, $\left.\delta p / p=0.1 \%\right)$

Circulating beam (Run LEB018A):

$11.039 \mathrm{~mm}^{*} 7.074 \mathrm{~mm}$, half size, at the entrance of the septum magnet, $12.013 \mathrm{~mm} * 6.374 \mathrm{~mm}$, half size, at the exit.

Injection beam (Run LEB018C and LEB630E):

$11.028 \mathrm{~mm}^{*} 7.074 \mathrm{~mm}$, half size, at the entrance of the septum magnet,

$12.011 \mathrm{~mm} * 6.374 \mathrm{~mm}$, half size, at the exit.

(2) Separations between the two centroids of circulating beam and injection beam (Run LEB018C and LEB630E)

Horizontal separations:

$34.394 \mathrm{~mm}$, at the entrance of the septum magnet,

$37.219 \mathrm{~mm}$, at the exit.

Vertical separations:

$37.928 \mathrm{~mm}$, at the entrance of the septum magnet,

$0.000 \mathrm{~mm}$, at the exit. 
The geometry of the Lambertson septum magnet is so arranged that the mid-plane of the magnet gap is parallel with the central trajectory plane of the injection beam. The minimum edge-to-edge separation of the two beams in the horizontal direction (from the right edge of the circulating beam to the left edge of the injection beam) is $11.344 \mathrm{~mm}$, as shown in Figure 6. If the beam centroid displacement caused by magnet errors is $3.0 \mathrm{~mm}$, the distance from the right edge of the circulating beam to the vertex of the field-free region has to be at least $6.315 \mathrm{~mm}$, as shown in Figure 7. In this case, the space available for septum is $2.029 \mathrm{~mm}$ (Figure 6), it meets the requirement.

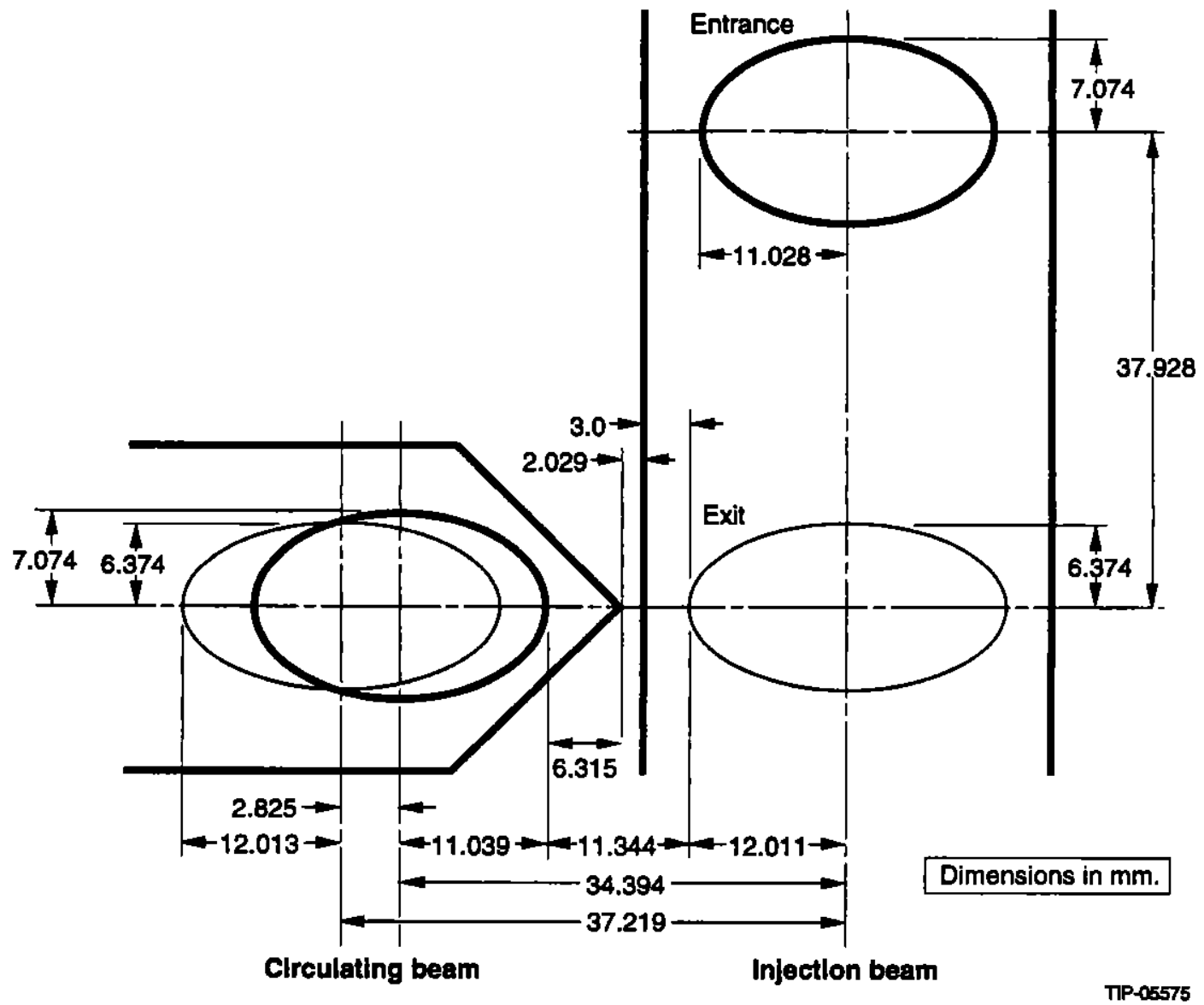

Figure 6. Beam Parameters in the Septum Magnet. 


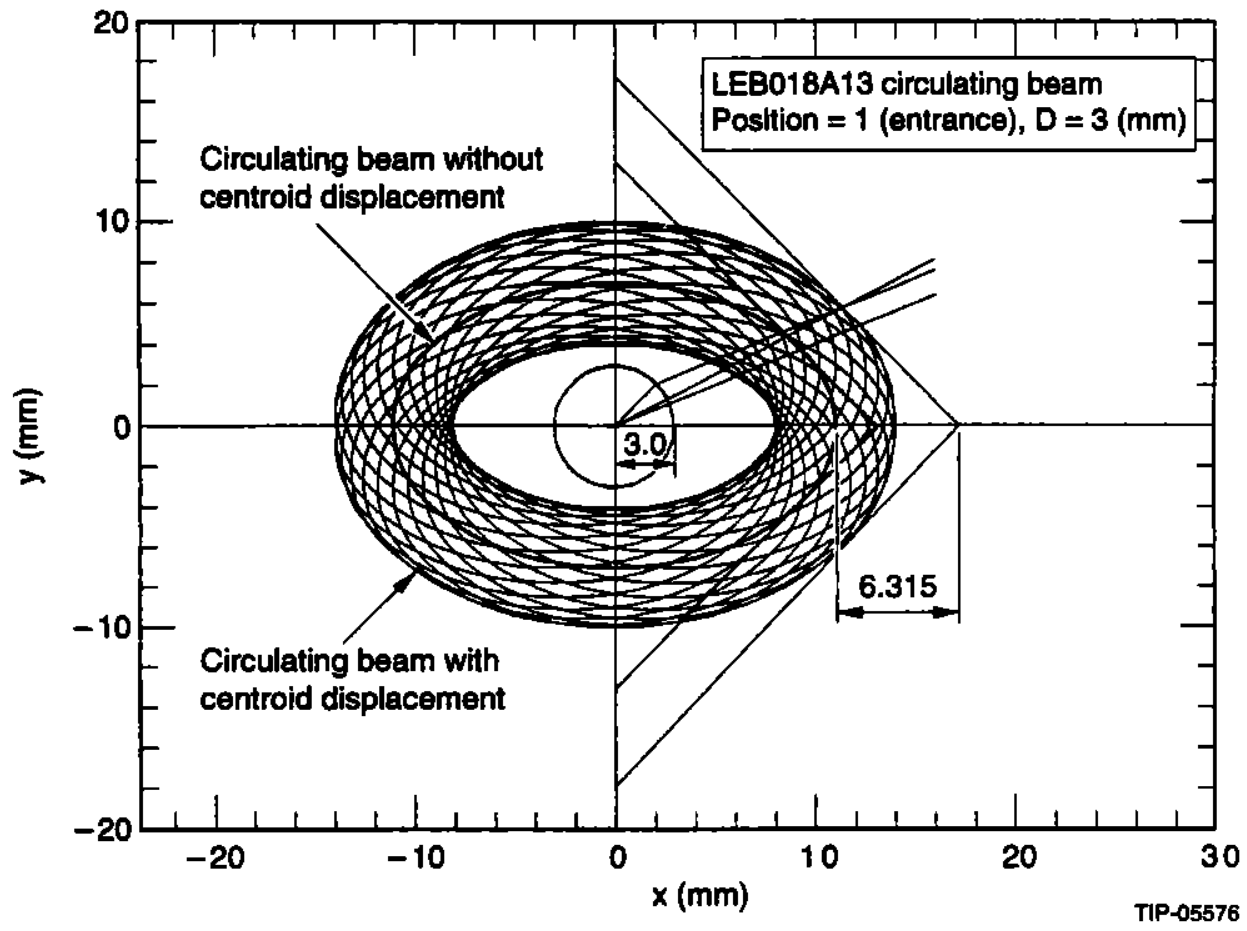

Figure 7. Circulating Beam with or without Centroid Displacement Caused by Magnet Errors.

\subsection{PARAMETERS OF SEPTUM MAGNET AND INJECTION KICKER}

Based on the beam optics design of the MEB injection system, the main parameters of the injection Lambertson septum magnet are determined as follows: ${ }^{4}$

Number

Field aperture $\left(w^{*} h\right)$

Field-free aperture $\left(w^{*} h\right)$

Leakage field in field-free region (max, at the center of circulating beam)

Magnet length

Good field region $\left(w^{*} h\right)$

Field (max)

Field quality ( $\triangle \mathrm{BL} / \mathrm{BL}$ )

TOD of power supply
1

$>60 \mathrm{~mm}^{*} \pm 15 \mathrm{~mm}$

$50.8 \mathrm{~mm} * 70 \mathrm{~mm}$

$<0.0008 \mathrm{~T}$

$<0.02 \mathrm{~T} / \mathrm{m}$

$<0.2 \mathrm{~T} / \mathrm{m}^{2}$

$2.0 \mathrm{~m}$

$60 \mathrm{~mm}^{*} \pm 15 \mathrm{~mm}$

$0.78 \mathrm{~T}$

$1 * 10^{-4}$

$1 * 10^{-4}$ 
Here, $w$ and $h$ are with respect to the magnet gap, $h$ is measured in the gap height direction. The conceptual design of the Lambertson septum magnet is shown in Figure $8 .^{5}$

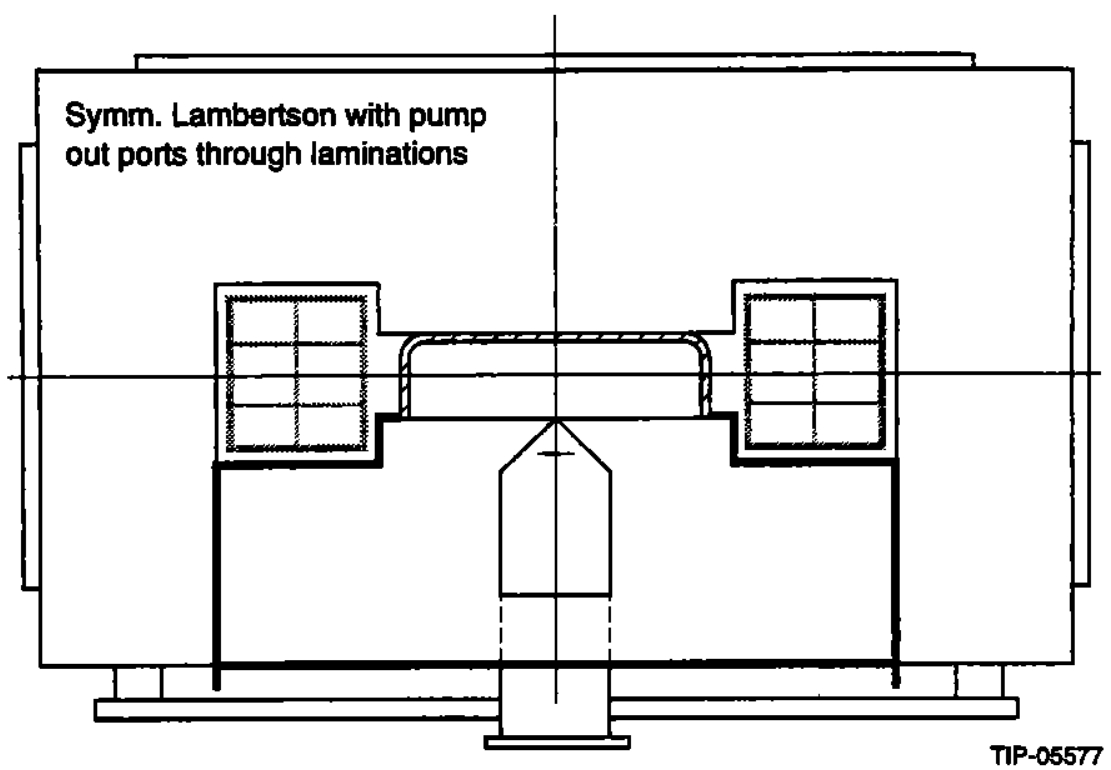

Figure 8. Conceptual Design of the Lambertson Septum Magnet.

The requirements for the injection kickers are as follows:

$\begin{array}{ll}\text { Number of magnets } & \geq 4 \\ \text { Slot length } & 6.45 \mathrm{~m} \\ \text { Aperture }\left(\mathrm{H}^{*} \mathrm{~V}\right) & 100 \mathrm{~mm} * 50 \mathrm{~mm} \\ \text { Strength (bending angle) } & 0.90 \mathrm{mrad} \\ \text { BL } & 0.036 \mathrm{~T}^{*} \mathrm{~m} \\ \text { TOD of power supply } & 1 * 10^{-2}\end{array}$

A twelve magnets kicker system design has been reported, ${ }^{6}$ where each magnet consists of ten cells. 


\section{REFERENCES}

1. "Element Specification (Level 3A) for the Medium Energy Booster Accelerator of the Superconducting Super Collider," No. E10-000022, WBS 231, Rev. B, Superconducting Super Collider Laboratory, 1993.

2. N. Mao et al., "Beam Optics of LEB-MEB Transfer Line for Superconducting Super Collider," Proceedings of the 1993 Particle Accelerator Conference, Washington, D.C., p. 333.

3. K. Brown et al., "TRANSPORT, a Computer Program for Designing Charged Particle Beam Transport Systems," SLAC-91, Rev. 3, 1993.

4. "Element Specification (Level 3B) for the Low Energy Booster to Medium Energy Booster (LEB-MEB) Beam Transfer Line of the Superconducting Super Collider," No. E10-000030, WBS 222, Rev. D, Superconducting Super Collider Laboratory, 1994.

5. S. Sheynin, "Conceptual Design-Type B Symmetry Lambertson," Superconducting Super Collider Laboratory, 1993.

6. D. Anderson, "MEB Kicker Magnet System Design Status," Superconducting Super Collider Laboratory, July 1993. 